\title{
Desenvolvimento dental e idade cronológica em pacientes com fissuras labiopalatinas: uma revisão de literatura
}

\author{
Dental development and chronological age in patients with cleft lip and palate: a literature review \\ Desarrollo odontológico y de la edad cronológica en pacientes con fisuras labiopalatinas: una revisión de literatura \\ Andressa Cavalcanti PIRES ${ }^{1}$ \\ Marina Tavares Costa NÓBREGA ${ }^{1}$ \\ Tânia Braga RAMOS ${ }^{2}$ \\ Rosa Helena Wanderley LACERDA \\ ${ }^{I}$ Doutoranda do Programa de Pós-Graduação em Odontologia, Departamento de Odontologia, Universidade Estadual da Paraíba, UEPB \\ 58429-500, Campina Grande - PB, Brasil \\ ${ }^{2}$ Professora Mestre do Curso de Especialização em Ortodontia da ABO-PB, 58040-490, João Pessoa-PB, Brasil
}

\begin{abstract}
Resumo
Introdução: A idade dentária representa um aliado na avaliação da idade fisiológica, que comparada a idade cronológica, podem orientar quanto as alterações de desenvolvimento. Objetivo: Avaliar o atraso de desenvolvimento dental relacionado a idade cronológica do indivíduo portador de fissura labiopalatina. Material e método: Para realização da revisão da literatura foi utilizada abordagem indutiva e técnica documental baseada na literatura pré-existente sobre o assunto. A pesquisa abrange a busca de artigos publicados nas bases de referências bibliográficas PUBMED, Portal de Periódicos CAPES, SCIELO e BIREME. Utilizou-se como descritores: "odontogênese", "fissura labial", "fissura palatina", "odontogenesis", "cleft lip" e "cleft palate". Resultados: Após excluídas as duplicatas, 34 artigos foram encontrados nas bases de dados selecionadas. Sendo 32 na base Pubmed, 25 na base Bireme, 04 no Portal de Periódicos CAPES e nenhum na base Scielo. Destes, 04 foram selecionados para análise. Conclusão: Pode-se concluir com esse trabalho que existe um atraso no desenvolvimento dentário de pacientes com fissura lábiopalatina em relação aos pacientes não fissurados e um atraso no desenvolvimento dentário de 6 meses deve ser considerado ao planejar o tratamento e cirurgias. É inconclusiva a diferença relacionada ao gênero.
\end{abstract}

Descritores: Odontogênese; Fissura Labial; Fissura Palatina.

\section{Abstract}

Introduction: Dental age is a component in the evaluation of physiological age, which compares the chronological age, can be oriented for developmental changes. Objective: to evaluate the delay of dental development related to a chronological age of the individual with cleft lip and palate. Material and method: For the accomplishment of the study of revision an inductive and documentary technique based on the preexisting literature on the subject was used. The research encompasses a search of articles published in the bibliographic reference bases PUBMED, Portal of Periodicals CAPES, SCIELO and BIREME. Descriptors: "odontogenesis", "cleft lip" and "cleft palate". Results: After being excluded as duplicates, 34 were found in the selected databases. Being 32 in the Pubmed database, 25 in the Bireme database, 04 in the Portal of CAPES Journals and none in the Scielo database. Of these, 04 were selected for analysis. Conclusion: One can conclude with the work that exists in the delay in the development of a patient with cleft lip and palate and the relationship with the patients is not a problem in the dental development of six months. The difference related to gender is inconclusive.

Descriptors: Odontogenesis; Cleft Lip; Cleft Palate.

\section{Resumen}

Introducción: La edad dental es un componente en la evaluación de la edad fisiológica, que compara la edad cronológica, puede ser orientado en cuanto a cambios de desarrollo. Objetivo: evaluar el retraso de desarrollo odontológico relacionado a una edad cronológica del individuo portador de fisura labiopalatina. Material y método: Para la realización del estudio de revisión se utilizó un enfoque inductivo y técnico documental basado en la literatura preexistente sobre el tema. La investigación abarca una búsqueda de artículos publicados en las bases de referencia bibliográficas PUBMED, Portal de Periódicos CAPES, SCIELO y BIREME. Descirptores: "odontogénesis", "fisura labial", "fisura palatina", "odontogenesis", "cleft lip" y "cleft palate". Resultados: Después de excluidos como duplicados, 34 se encontraron en las bases de datos seleccionadas. Siendo 32 en la base Pubmed, 25 en la base Bireme, 04 en el Portal de Periódicos CAPES y ninguno en la base Scielo. De estos, 04 fueron seleccionados para análisis. Conclusión: Se puede concluir con el trabajo que existe en el retraso en el desarrollo de un paciente con fisura labiopalatina y la relación con los pacientes no es un problema en el desarrollo dental de seis meses. Es inconclusa la diferencia relacionada al género.

Descriptores: Odontogénesis; Labio Leporino; Fisura del Paladar.

\section{INTRODUÇÃO}

Durante a vida intrauterina inicia-se $o$ processo de desenvolvimento dos dentes, a partir da sexta semana começa a formação da lâmina dental, no quarto mês observamos os primeiros sinais de calcificação dos dentes decíduos e no sexto mês todos os dentes decíduos já iniciaram seu desenvolvimento. A calcificação do primeiro molar permanente tem seu início no quinto mês de vida intrauterina ${ }^{1}$.

A idade dentária representa um aliado na avaliação da idade fisiológica, que comparada à idade cronológica, pode orientar quanto as alterações de desenvolvimento. Sendo necessário conhecer o tempo médio em que cada dente deve irromper ${ }^{2}$. É importante salientar que muitos fatores podem alterar a cronologia de erupção, pelas variações genéticas na ordem de aparecimento dos centros de ossificação e formação dentária, tais como alimentação, grupo étnico, clima, gênero, fatores sócio econômicos, entre outros $^{1,3}$

O equilíbrio fisiológico influencia diretamente na evolução normal da dentição e uma alteração orgânica pode acelerar ou atrasar o tempo que os dentes irrompem na cavidade bucal 4 . Considerando fatores sistêmicos, as fissuras labiopalatinas estão associadas a muitas alterações que modificam o estado de saúde ${ }^{5}$.

As fissuras labiopalatinas são malformações do terço médio da face que ocorrem no período embrionário, a partir da quarta semana de vida intrauterina, decorrentes da falta de fusão dos processos nasais mediais entre si, e destes com os processos maxilares laterais. Estudos mostram que as causas podem ser multifatoriais, envolvendo causas 
ambientais e genéticas, constituindo um problema médico-odonto-social ${ }^{6,7}$.

Essas alterações são as mais comuns das estruturas orofaciais e ocorrem com uma prevalência que pode variar de um para cada 500 até um para cada 2500 nascidos vivos ${ }^{8-14}$.

$\mathrm{Na}$ literatura existem trabalhos ${ }^{15}$ que relatam um atraso na formação dentária de pacientes com fissura labiopalatina quando comparados a pacientes não portadores de fissura, levando a uma erupção também atrasada. Porém, os resultados são inconclusivos em relação a etiopatogenia que ocorre esse atraso. O presente estudo objetiva realizar uma revisão de literatura a respeito desse atraso de desenvolvimento dental relacionado à idade cronológica do indivíduo portador de fissura labiopalatina.

\section{MATERIAL E MÉTODO}

Para realização do estudo foi utilizada uma abordagem indutiva e técnica documental baseada na literatura pré-existente sobre o assunto ${ }^{16}$. A pesquisa abrange a busca de artigos publicados nas bases de referências PUBMED, Portal de Periódicos CAPES, SCIELO e BIREME. Utilizou-se como descritores: "odontogênese", "fissura labial", "fissura palatinal", "odontogenesis", "cleft lip" e "cleft palate".

Como critérios de elegibilidade, foram incluídos os artigos publicados entre os anos de 2003 a 2018, escritos em inglês e português que possuiam o texto completo disponível dentro da temática abordada. Foram excluídos os artigos que não abordavam o objetivo desta revisão e aqueles realizados em animais.

\section{RESULTADOS E DISCUSSÃO}

Excluídas as duplicatas, 34 artigos foram encontrados nas bases de dados selecionadas. Sendo 32 na base Pubmed, 25 na base BIREME, 04 no Portal de Periódicos CAPES e nenhum na base SCIELO. Destes, 04 foram selecionados para análise de acordo com os critérios de inclusão, como pode ser observado no Quadro 1.

De acordo com o Quadro 1, os artigos relacionaram grupos pareados entre crianças com fissura labiopalatina e crianças sem fissura para avaliação, o que ajuda a minimizar fatores de confusão. Nos estudos de Borodkin et al. ${ }^{17}$, Lai et al. ${ }^{18}$ e Tan et al. ${ }^{19}$, o método de Demirjian é citado por sua precisão em estimar idade dental, particularmente durante a primeira infância. No estudo de Celebi et al. ${ }^{20} \mathrm{o}$ volume dentário dos incisivos centrais superiores é avaliado através de tomografia computadorizada de feixe cônico.

No estudo de Lai et al. ${ }^{18}$ as crianças com idades entre 3 e 12 anos, com e sem fissura de lábio e palato, foram pareadas por idade e gênero (231 ao todo). Ambos os grupos, com e sem fissura, tinham idades dentárias que eram avançadas as suas idades cronológicas, e isso foi maior em crianças não fissuradas. Borodkin et al. ${ }^{17}$ avaliaram crianças entre 6 e 13 anos de idade, sendo 49 crianças com fissuras e 49 controles pareados. E observaram que seu estudo não forneceu crianças suficientes em cada idade para concluir sobre atrasos e diferentes idades cronológicas, mas um atraso no desenvolvimento de 6 meses deve ser considerado ao planejar o tratamento e cirurgias. Tan et al. ${ }^{19}$ realizaram um estudo de caso-controle com crianças, de 9 a 13 anos, com fissura labiopalatina unilateral completa e controles completos (crianças sem fissura pareadas em idade, gênero e etnia). Constataram que o atraso na maturação dentária era atenuado com a idade.

\begin{tabular}{|c|c|}
\hline \multicolumn{2}{|c|}{ Borodkin et al. $2008^{17}$} \\
\hline Título & Permanent tooth development in children with cleft lip and palate \\
\hline Amostra & $\begin{array}{l}\text { Crianças entre } 6 \text { e } 13 \text { anos de idade. } 49 \text { crianças com fissuras e } 49 \text { controles } \\
\text { pareados. }\end{array}$ \\
\hline Resultados & $\begin{array}{l}\text { Análises indicaram uma correlação entre o atraso no desenvolvimento do dente } \\
\text { permanente e a fissura de lábio e palato, com um atraso total de o,52 anos ( } \mathrm{P}= \\
\text { o,o2) e com os meninos respondendo por todo o atraso. Não foram encontradas } \\
\text { diferenças entre indivíduos com fissuras unilaterais ou bilaterais. Uma tendência } \\
\text { nấ significativa foi notada para maior atraso em indivíduos com fissuras dos } \\
\text { palatos primário e secundário vs palato primário sozinho. }\end{array}$ \\
\hline Conclusão & $\begin{array}{l}\text { Embora o desenvolvimento permanente dos dentes esteja atrasado em pacientes } \\
\text { com fissura labiopalatina, esse atraso é: encontrado apenas em meninos; é } \\
\text { independente do tipo e gravidade da fenda; e não está correlacionado com a } \\
\text { proximidade da fissura. }\end{array}$ \\
\hline \multicolumn{2}{|c|}{ Lai et al. $2008^{18}$} \\
\hline Título & Dental development of Chinese children with cleft lip and palate \\
\hline Amostra & $\begin{array}{l}\text { Pares pareados por idade e gênero ( } 231 \text { ao todo) de crianças chinesas do sul com } \\
\text { idades entre } 3 \text { e } 12 \text { anos, com e sem fissura de lábio e palato. }\end{array}$ \\
\hline Resultados & 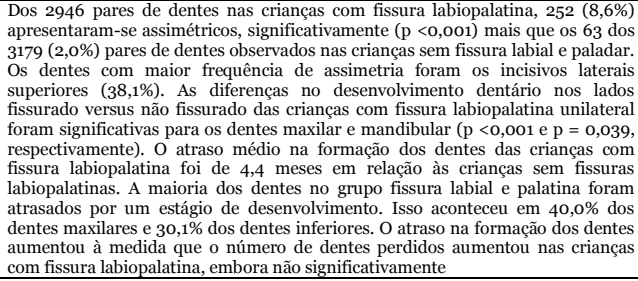 \\
\hline Conclusão & $\begin{array}{l}\text { Esse grupo de crianças do sul da China com fissura labiopalatina demonstrou } \\
\text { maior prevalência de desenvolvimento dentário assimétrico e tardio do que suas } \\
\text { contrapartes que não apresentavam fissura labiopalatina. }\end{array}$ \\
\hline \multicolumn{2}{|c|}{ Tan et al. $2017^{19}$} \\
\hline Título & $\begin{array}{l}\text { Longitudinal dental maturation of children with complete unilateral cleft lip and } \\
\text { palate: A case-control cohort study }\end{array}$ \\
\hline Amostra & $\begin{array}{l}115 \text { radiografias de crianças de } 9 \text { a } 13 \text { anos, com fissura labiopalatina unilateral } \\
\text { completa e controles completos (crianças não-CLP pareadas em idade, gênero e } \\
\text { etnia) de uma clínica odontológica em Cingapura }\end{array}$ \\
\hline Resultados & $\begin{array}{l}\text { A maturação dental tardia foi encontrada em crianças com } 5 \text { a } 9 \text { anos de idade } \\
\text { com fissura em comparação com os controles em o,55 anos (desvio padrão: } 0,75) \\
\text { (P <o,oo1). Não houve diferença significativa entre a maturação dental de } \\
\text { crianças com fissura e controles no grupo de } 9 \text { a } 13 \text { anos de idade }(\mathrm{P}=0,744) \text {. }\end{array}$ \\
\hline Conclusão & $\begin{array}{l}\text { Não houve diferença na maturação dentá́ria entre fissurados e controles no grupo } \\
\text { de } 9 \text { a } 13 \text { anos de idade. No entanto, houve diferença diametral na maturação } \\
\text { dentária no grupo de } 5 \text { a } 9 \text { anos de idade, que atenuou à medida que cresciam. } \\
\text { Houve um risco consistentemente maior de formaçãa de dentes assimétricos em } \\
\text { crianças com fissuras do que nos controles. }\end{array}$ \\
\hline \multicolumn{2}{|c|}{ Celebi et al. $2015^{20}$} \\
\hline Título & $\begin{array}{l}\text { Effects of cleft lip and palate on the development of permanent upper central } \\
\text { incisors: a cone-beam computed tomography study }\end{array}$ \\
\hline Amostra & $\begin{array}{l}40 \text { pacientes ( } 20 \text { homens e } 20 \text { mulheres; média de idade: } 14,84 \pm 3,2 \text { anos) com } \\
\text { pacientes não-sindrômicos e } 40 \text { pacientes-controle ( } 20 \text { homens e } 20 \text { mulheres; } \\
\text { média de idade: } 13,38 \pm 1,6 \text { anos). }\end{array}$ \\
\hline Resultados & 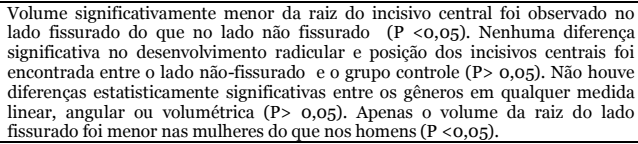 \\
\hline Conclusão & $\begin{array}{l}\text { Em geral, os volumes das raizes dos incisivos centrais no lado da fenda foram } \\
12,15 \% \text { menores do que o lado não fissurado. O desenvolvimento da raiz do } \\
\text { incisivo central foi muito mais influenciado pela fissura nas mulheres do que nos } \\
\text { homens. }\end{array}$ \\
\hline
\end{tabular}

Celebi et al. $^{20}$ afirmaram que o desenvolvimento radicular de incisivos superiores foram afetados pela fissura, não havendo diferenças significativas no volume do incisivos centrais entre o lado não fissurado em pacientes com grupo de controle, mostrando que os fatores ambientais desempenham um papel na determinação do volume da raiz, em vez da genética.

Lai et al. ${ }^{18}$ não observaram diferença significativa entre os gêneros corroborando o encontrado por Ranta ${ }^{21}$. No entanto, observa mais 
retardos no desenvolvimento dentário em meninas com fissura labiopalatina do que em meninas sem fissura. Deste modo, além de um fator local relacionado à formação de fissuras, existia um fator mais generalizado, como genética, que foi responsável pelo atrasado no desenvolvimento dental entre as crianças com fissura labiopalatina. Atribuindo a assimetria encontrada em pares de dentes a fatores genéticos e, possivelmente, em alguns casos, aos procedimentos cirúrgicos para reparar o defeito de fenda.

Já Borodkin et al. ${ }^{17}$ relataram que os meninos com a fenda mostraram um atraso estatisticamente significativo em relação aos sem fissura. E que as meninas apresentaram um desenvolvimento dental mais acelerado que os meninos, fazendo com que o atraso não seja percebido nas meninas com fissura. Tan et al. ${ }^{19}$ concluem que não houve diferença estatisticamente significante entre gênero, nem diferenças étnicas em relação ao desenvolvimento dentário.

Celebi et al. ${ }^{20}$ estudaram os dentes incisivos centrais superiores para avaliar desenvolvimento da raiz nos pacientes fissurados por serem os primeiros dentes erupcionados ao redor da fissura. Incisivos laterais são comumente ausentes ou têm malformações de raiz e caninos são os últimos da dentição permanente a entrar em erupção e completar o desenvolvimento da raiz. Observaram também que o desenvolvimento radicular dos incisivos centrais superiores do lado não fissurado não foi afetado pelo gênero. Pelo contrário, o volume da raiz dos incisivos centrais superiores no lado fissurado foi encontrado menor em mulheres.

Borodkin et al. ${ }^{17}$ ao comparararem os atrasos no desenvolvimento dentário entre os sujeitos com diferentes tipos de fissuras (unilaterais versus bilaterais) não encontraram diferenças significativas. Os incisivos laterais foram os mais atrasados, seguido pelos caninos e pré-molares.

Celebi et al. ${ }^{20}$ concluíram que o volume radicular do incisivo central no lado fissurado foram $12,15 \%$ menores em comparação ao lado não fissurado.

Os achados nos mostram que há um atraso no desenvolvimento dental, mas que existem muitas lacunas a serem preenchidas para que esses resultados sejam extrapolados para população, por serem contraditórios.

\section{CONCLUSÃO}

Apesar do pequeno número de artigos encontrados na literatura, a presente revisão sugere um atraso no desenvolvimento dentário de pacientes com fissura lábiopalatina em relação aos pacientes não fissurados e um atraso no desenvolvimento dentário de 6 meses deve ser considerado ao planejar o tratamento e cirurgias. É inconclusiva a diferença relacionada ao gênero. Conclui-se, portanto, a necessidade de novos estudos que venham a confirmar a hipótese sugerida, bem como para compreensão dos fatores etiológicos que envolvem esta alteração de desenvolvimento dentário.

\section{REFERÊNCIAS}

1. Vellini-Ferreira F. Ortodontia: Diagnóstico e Planejamento Clínico. 7. ed. São Paulo: Artes Médicas Ltda; 2008.

2. Carrara CFC. Estudo da cronologia e sequência de erupção e das agenesias dos dentes permanentes em indivíduos brasileiros, leucodermas, portadores de fissura transforame incisivo unilateral [dissertação]. Bauru: Faculdade de Odontologia de Bauru - USP; 2000.

3. Carvalho AAF, Carvalho A, Santos Pinto MC. Estudo radiográfico do desenvolvimento da dentição permanente de crianças brasileiras com idade cronológica variando entre 84 e 131 meses. Rev. Odonto UNESP. 1990;19:(1):31-9.

4. Toledo OA. Aspectos da cronologia de erupção dos dentes permanentes. Considerações sobre o efeito da urbanização na alteração da cronologia eruptiva. Rev. Odontol Araçatuba.1965;1:47-64.

5. Loevy HT, Aduss H. Tooth maturation in cleft lip, cleft palate, or both. Cleft Palate J.1988; 25(4):343-47.

6. Ellis III. Management of Patients with Orofacial Clefts. In: Hupp, Ellis III, Tucker. Contemporary oral and maxillofacial surgery, $6^{\mathrm{a}}$ th. Misssouri: Elsevier; 2014.

7. Freitas e Silva DS, Mauro LDL, Oliveira LB, Ardenghi TM, Bönecker M. Estudo descritivo das fissuras lábio-palatinas relacionadas a fatores individuais, sistêmicos e sociais. RGO. 2008;56(4):387-91.

8. Conway JC, Taub PJ, King R, Oberoi K, Doucette $\mathrm{J}$, Jabs EW. Ten-year experience of more than 35,000 orofacial clefts in Africa. BMC Pediatr. 2015; $15: 8$.

9. Faraj JORA, André M. Alterações dimensionais transversas do arco dentário com fissura labiopalatina, no estágio de dentadura decídua. $\mathrm{R}$ Dental Press Ortodon Ortop Facial. 2007; 12(5):100-8.

10.Silva Filho OG, Freitas JAS. Caracterização Morfológica e Origem Embriológica. In: Trindade IEK, Silva Filho OG (orgs). Fissuras labiopalatinas: uma abordagem interdisciplinar. São Paulo: Santos; 2007.p.17-49.

11.Watson ACH. Embriologia, etiologia e incidência. In: Watson ACH, Sell DA, Grunwell P (orgs). Tratamento de fissura labial e fenda palatina. São Paulo: Santos; 2005.p.3-15

12.Lages EMB, Marcos B, Pordeus IA. Oral health of individuals with cleft lip, cleft palate, or both. Cleft Palate-Craniofac J. 2004;41(1):59-63. 
13.Zandi M, HeidarI A. An epidemiologic study of orofacial clefts in Hamedan city, Iran: a 15-year study. Cleft Palate-Craniofac J. 2011;48(4 ):483-89.

14. Coutinho ALF, Lima MC, Kitamura MAP, Ferreira Neto J, Pereira RM. Perfil epidemiológico dos portadores de fissuras orofaciais atendidos em um Centro de Referência do Nordeste do Brasil. Rev. Bras Saúde Mater Infant. 2009;9(2):149-56.

15.Tan EL, Yow M, Kuek MC, Wong HC. Dental maturation of unilateral cleft lip and palate. Ann Maxillofac Surg. 2012;2(2):158-62.

16.Lakatos EM, Marconi MA. Fundamentos de metodologia científica. 7 ed. São Paulo: Atlas, 2010.

17.Borodkin AF, Feigal RJ, Beiraghi S, Moller KT, Hodges JS. Permanent tooth development in children with cleft lip and palate. Pediatr Dent. 2008; 30:408-13.

18.Lai MC, King NM, Wong HM. Dental development of Chinese children with cleft lip and palate. Cleft Palate Craniofac J. 2008; 45:289-96.

19.Tan ELY, Kuek MC, Wong HC, Yow M. Longitudinal dental maturation of children with complete unilateral cleft lip and palate: a casecontrol cohort study.Orthod Craniofac Res.2017; 20(4):189-95.

20.Celebi AA, Ucar FI, Sekerci AE, Caglaroglu M, Tan E. Effects of cleft lip and palate on the development of permanent upper central incisors: a cone-beam computed tomography study. Eur $\mathbf{J}$ Orthod. 2015; 37(5):544-49.

21.Ranta R. A comparative study of tooth formation in the permanent dentition of Finnish children with cleft lip and palate. Proc Fin Dent Soc. 1972;68(2):58-66.

\section{CONFLITO DE INTERESSES}

Os autores declaram não haver conflitos de interesse.

\section{AUTOR PARA CORRESPONDENCIA}

Andressa Cavalcanti Pires

andressa_cavalcanti@hotmail.com

Submetido em 15/10/2018

Aceito em 12/03/2019 